TRANSACTIONS OF THE

AMERICAN MATHEMATICAL SOCIETY

Volume 351, Number 7, Pages 2927-2948

S 0002-9947(99)02344-2

Article electronically published on March 29, 1999

\title{
ROTATION AND ENTROPY
}

\author{
WILLIAM GELLER AND MICHAE MISIUREWICZ
}

\begin{abstract}
For a given map $f: X \rightarrow X$ and an observable $\varphi: X \rightarrow \mathbb{R}^{d}$, rotation vectors are the limits of ergodic averages of $\varphi$. We study which part of the topological entropy of $f$ is associated to a given rotation vector and which part is associated with many rotation vectors. According to this distinction, we introduce directional and lost entropies. We discuss their properties in the general case and analyze them more closely for subshifts of finite type and circle maps.
\end{abstract}

\section{INTRODUCTION}

The notions of rotation vectors (or numbers) and rotation sets used here are fairly general. We have a dynamical system, that is a map $f: X \rightarrow X$, and an observable (that is a function $\varphi: X \rightarrow \mathbb{R}^{d}$ ), and we look at the ergodic averages at various points. If these averages converge at a given point, the limit is the rotation vector at this point. The set of all rotation vectors is the rotation set. When describing this, we speak of the rotation theory. If $\varphi$ is the displacement function (measuring the vector by which the point is displaced in the universal covering space), we get the classical rotation sets. However, this formalism can also be applied to various other physical and mathematical (see e.g. [BM]) situations.

For dynamical systems with some rotation theory a natural question arises: how is topological entropy connected with this rotation theory? In particular, how large is the entropy of the part of the dynamics that is non-trivial from the point of view of "rotations"? How large is the entropy of the part of the dynamics that is connected with one particular rotation number (vector)?

There have been some attempts to answer these questions, and in particular to define "rotational entropy". One such attempt, in [Bot], has been unsuccessful, as we show in Section 2. Another such notion, defined in [Sw] and [KS], although useful, measures something else. In particular, it is not a topological invariant and can be larger than the topological entropy. A notion of topological entropy at a given rotation vector has been introduced in $[\mathrm{Kw}]$. Although it serves its purpose well, we do not adopt it, since we do not see any possibility of defining a complementary notion of rotational entropy.

To define our notions of "rotational" and "directional" entropies, we employ the variational principle. Since topological entropy is the supremum of metric entropies of ergodic invariant measures, we classify these measures as either connected with

Received by the editors February 22, 1997.

1991 Mathematics Subject Classification. Primary 54H20, 58F99, 58F11.

Key words and phrases. Rotation sets, entropy.

The second author was partially supported by NSF grant DMS-9305899.

(C)1999 American Mathematical Society 
some rotation vector (directional measures) or not (lost measures). Then the directional entropy in a given direction and the lost entropy are defined as suprema of measure entropies over appropriate classes of measures. We use the term "lost" in order to distinguish our lost entropy from other kinds of rotational entropies. After all, without any direction it is surely lost.

We prove (in Section 4) that these notions can be defined also in a purely topological manner. Then, in Sections 5 and 6 , we study more closely the cases of subshifts of finite type and circle maps, respectively. The case of subshifts of finite type can be applied in many situations when the system admits a Markov partition (see e.g. Section 2 of $[\mathrm{Z}]$ ).

\section{Rotational ENTROPY VANishes}

We begin by considering the notion of rotational entropy for the annulus proposed in [Bot]. We show that the invariant defined there vanishes for all orientation preserving annulus homeomorphisms, in fact for all continuous maps homotopic to the identity.

Let $A=S^{1} \times[0,1]$ be the annulus and $f: A \rightarrow A$ a continuous map homotopic to the identity. Then the universal cover of $A$ is the strip $S=\mathbb{R} \times[0,1]$ and we let $p: S \rightarrow A$ be the usual covering map given by exponentiation on the first coordinate. Choose a lifting $F: S \rightarrow S$ of $f$. For $x \in A$ we write $\bar{x}$ for the preimage $p^{-1}(x) \cap[0,1) \times[0,1]$, and we take $F_{1}^{l}: S \rightarrow \mathbb{R}$ to be the composition of $F^{l}$ with projection $\pi_{1}$ onto the first coordinate of $S$ (if $l=0$, we write just $F_{1}$ ). We will also write $\bar{x}_{1}$ for $\pi_{1}(\bar{x})$.

Following [Bot], call a subset $E$ of the annulus $(n, \varepsilon)$-rotational spanning if for all $x \in A$ there exists $y \in E$ with $\|\bar{x}-\bar{y}\|<\varepsilon$ and $\left|F_{1}^{j}(\bar{x})-F_{1}^{j}(\bar{y})\right| / j \leq \varepsilon$ for $j=1,2, \ldots, n-1$. The $\varepsilon$-rotational entropy is $h_{r, \varepsilon}(f)=\limsup _{n \rightarrow \infty}(1 / n) \log s(n, \varepsilon)$, where $s(n, \varepsilon)$ is the minimal cardinality of an $(n, \varepsilon)$-rotational spanning set. Then the rotational entropy is $h_{r}(f)=\lim _{\varepsilon \rightarrow 0} h_{r, \varepsilon}(f)$.

The observable $\varphi$ that we are working with is given by $\varphi(x)=F_{1}(\bar{x})-\bar{x}_{1}$ on the annulus. We will write $\varphi(l, x)$ for $\sum_{i=0}^{l-1} \varphi\left(f^{i}(x)\right)=F_{1}^{l}(\bar{x})-\bar{x}_{1}$. There exists a constant $M$ such that for all $x$ in $A,|\varphi(1, x)| \leq M$. Then $|\varphi(l, x)| \leq M l$ for every $x \in A$ and $l \geq 1$.

Fix $\delta, N$, and $m$. We will call a set $B \subset A k$-small if for every $x, y \in B$ we have:

1. $\|\bar{x}-\bar{y}\| \leq \delta$,

2. $\left|F_{1}^{j}(\bar{x})-F_{1}^{j}(\bar{y})\right| / j \leq \delta$ for $j=1,2, \ldots, N$,

3. $\left|\varphi\left(m, f^{N+i m}(x)\right)-\varphi\left(m, f^{N+i m}(y)\right)\right| / m \leq \delta$ for $i=0,1, \ldots, k$.

Our first lemma constructs $(n, \varepsilon)$-rotational spanning sets from partitions of the annulus into $k$-small sets.

Lemma 2.1. For every $\delta \in(0, \varepsilon)$ and every $m$ there exists $N$ such that if $\mathcal{A}$ is a partition of $A$ into $k$-small sets and $E$ is a subset of $A$ formed by choosing a point from every element of $\mathcal{A}$, then $E$ is $(n, \varepsilon)$-rotational spanning for $n=N+k m+m-1$.

Proof. To show that $E$ is $(n, \varepsilon)$-rotational spanning for $n$ as above, it is enough to show that for every $x \in A$ there is $y \in E$ such that $\|\bar{x}-\bar{y}\|<\varepsilon$ and $\left|F_{1}^{j}(\bar{x})-F_{1}^{j}(\bar{y})\right| / j<\varepsilon$ for $j=1,2, \ldots, N+k m+m-1$. Let $\mathcal{A}$ and $E$ be as in the statement of the lemma. If $x \in A$ then there is $y \in E$ such that $x$ and $y$ belong to the same element of $\mathcal{A}$. Thus, $\|\bar{x}-\bar{y}\| \leq \delta<\varepsilon$ and $\left|F_{1}^{j}(\bar{x})-F_{1}^{j}(\bar{y})\right| / j \leq \delta<\varepsilon$ 
for $j=1,2, \ldots, N$. Hence, it remains to show that $\left|F_{1}^{j}(\bar{x})-F_{1}^{j}(\bar{y})\right| / j<\varepsilon$ for $j=N+1, N+2, \ldots, N+k m+m-1$. For such $j$ there are $i \in\{0,1, \ldots, k\}$ and $l \in\{0,1, \ldots, m-1\}$ such that $j=N+i m+l$. Note that

$$
\begin{aligned}
F_{1}^{j}(\bar{x})-F_{1}^{j}(\bar{y}) & =\left[F_{1}^{N}(\bar{x})-F_{1}^{N}(\bar{y})\right]+\sum_{p=0}^{i-1}\left[\varphi\left(m, f^{N+p m}(x)\right)-\varphi\left(m, f^{N+p m}(y)\right)\right] \\
& +\left[\varphi\left(l, f^{N+i m}(x)\right)-\varphi\left(l, f^{N+i m}(y)\right)\right] .
\end{aligned}
$$

We have $\left|F_{1}^{N}(\bar{x})-F_{1}^{N}(\bar{y})\right| \leq N \delta$ and $\left|\varphi\left(m, f^{N+p m}(x)\right)-\varphi\left(m, f^{N+p m}(y)\right)\right| \leq$ $m \delta$ for $p=0,1, \ldots, i-1$. Moreover, $\left|\varphi\left(l, f^{N+i m}(x)\right)-\varphi\left(l, f^{N+i m}(y)\right)\right| \leq 2 M l$. Therefore

$$
\left|\frac{F_{1}^{j}(\bar{x})-F_{1}^{j}(\bar{y})}{j}\right| \leq \frac{N \delta+i m \delta+2 M l}{N+i m+l} \leq \frac{N \delta+i m \delta+2 M m}{N+i m} \leq \delta+2 M m / N .
$$

If $N>2 M m /(\varepsilon-\delta)$ then $\delta+2 M m / N<\varepsilon$, so $\left|F_{1}^{j}(\bar{x})-F_{1}^{j}(\bar{y})\right| / j<\varepsilon$, as we wanted.

We now find, for each $k$, a partition of the annulus into $k$-small sets and give a bound on the cardinality of the partition.

Lemma 2.2. Let $\delta, N$ and $m$ be given. Then there exists a constant $K$ such that for every $k$ there exists a partition $\mathcal{A}_{k}$ of $A$ into $k$-small sets, of cardinality not exceeding $K(2 M / \delta+1)^{k}$.

Proof. We will construct the partitions $\mathcal{A}_{k}$ by induction. By continuity, if a subset of $[0,1) \times[0,1]$ has sufficiently small diameter then its projection to $A$ is 0 -small. Therefore there exists a finite partition $\mathcal{A}_{0}$ of $A$ into 0 -small sets. Denote its cardinality by $K$.

Now given $\mathcal{A}_{k}$ we will construct $\mathcal{A}_{k+1}$. Set $\psi(x)=\varphi\left(m, f^{N+(k+1) m}(x)\right)$. If $B \in$ $\mathcal{A}_{k}$ then $\psi(B)$ is a subset of $[-M m, M m]$. Therefore $\psi(B)$ can be partitioned into fewer than $2 M / \delta+1$ sets of diameter $\delta m$ or smaller. The inverse image under $\psi$ of this partition is a partition of $B$ into $(k+1)$-small sets. Taking the union of such partitions over all $B \in \mathcal{A}_{k}$ we get a partition $\mathcal{A}_{k+1}$ of $A$ into $(k+1)$-small sets and the cardinality of $\mathcal{A}_{k+1}$ is smaller than $2 M / \delta+1$ times the cardinality of $\mathcal{A}_{k}$.

Theorem 2.3. Let $f$ be a continuous map of the annulus homotopic to the identity. Then $h_{r}(f)=0$.

Proof. Fix $\varepsilon>0$. Set $\delta=\varepsilon / 2$. By Lemmata 2.1 and 2.2, for every $m$ there exist $N$ and $K$ such that for every $k$ there is a set $E_{k}$ that is $(n, \varepsilon)$-rotational spanning for $n=N+k m+m-1$ (and therefore for all smaller $n$ ) and has cardinality not larger than $K(4 M / \varepsilon+1)^{k}$. Therefore

$$
h_{r, \varepsilon}(f) \leq \limsup _{k \rightarrow \infty} \frac{1}{N+k m} \log \left[K(4 M / \varepsilon+1)^{k}\right]=\frac{1}{m} \log (4 M / \varepsilon+1) .
$$

Since $m$ is arbitrary, we get $h_{r, \varepsilon}(f)=0$.

\section{Desired properties}

Let us consider what properties we would like the rotational entropy $h_{\text {rot }}$ to have. We assume that $X$ is a compact Hausdorff space, $f: X \rightarrow X$ a continuous map, and $\varphi: X \rightarrow \mathbb{R}^{d}$ a continuous function. If there will be more maps involved, 
we will use subscripts. For instance we will write $\varphi_{f}$ to denote the function $\varphi$ used with $f$. Since the rotational entropy depends not only on the map, but also on the function $\varphi$, we will write $h_{r o t}(f, \varphi)$.

Let us list first the most obvious properties, namely the ones analogous to the basic properties of the usual topological entropy.

1. If $Y$ is a closed invariant subset of $X$, then $h_{r o t}\left(\left.f\right|_{Y},\left.\varphi\right|_{Y}\right) \leq h_{\text {rot }}(f, \varphi)$.

2. If $g$ is a factor of $f$ (via $\pi$ ) and $\varphi_{f}=\varphi_{g} \circ \pi$, then $h_{\text {rot }}\left(g, \varphi_{g}\right) \leq h_{\text {rot }}\left(f, \varphi_{f}\right)$.

3. If $\Omega(f)$ is the set of nonwandering points of $X$, then $h_{\text {rot }}\left(\left.f\right|_{\Omega(f)},\left.\varphi\right|_{\Omega(f)}\right)=$ $h_{\text {rot }}(f, \varphi)$.

4. If $\varphi_{f^{n}}=\sum_{i=0}^{n-1} \varphi_{f} \circ f^{i}$, then $h_{\text {rot }}\left(f^{n}, \varphi_{f^{n}}\right)=n h_{\text {rot }}\left(f, \varphi_{f}\right)$.

Note that (2) implies that if $g$ is conjugate to $f$ via $\pi$ and $\varphi_{f}=\varphi_{g} \circ \pi$ then $h_{\text {rot }}\left(g, \varphi_{g}\right)=h_{\text {rot }}\left(f, \varphi_{f}\right)$.

The next two properties we would like the rotational entropy to have are also more or less obvious. We say that real functions $\varphi_{1}$ and $\varphi_{2}$ are cohomologous if there is a bounded real function $\zeta$ such that $\varphi_{2}=\varphi_{1}+\zeta-\zeta \circ f$.

(5) $h_{\text {rot }}(f, \varphi) \leq h(f)$.

(6) If $\varphi_{1}$ and $\varphi_{2}$ are cohomologous, then $h_{\text {rot }}\left(f, \varphi_{1}\right)=h_{\text {rot }}\left(f, \varphi_{2}\right)$.

Finally, we state some specific properties that embody the idea of the rotational entropy (up to now, the usual entropy or simply zero would do, too). If we look at torus (or annulus) homeomorphisms isotopic to the identity (which is our main motivation), we can see various types of horseshoes. A horseshoe can be local, or can wrap around the torus. At the symbolic level, we should think of the full two-sided shift on two symbols 0 and 1 (we will denote it by $\sigma_{2}: \Sigma_{2} \rightarrow \Sigma_{2}$ ), with functions $\varphi_{0}, \varphi_{1}: \Sigma_{2} \rightarrow \mathbb{R}$ defined by $\varphi_{0} \equiv 0$ and $\varphi_{1}\left(\ldots, x_{-1}, x_{0}, x_{1}, \ldots\right)=x_{0}$. We should have $h_{\text {rot }}\left(\sigma_{2}, \varphi_{0}\right)=0$ and $h_{\text {rot }}\left(\sigma_{2}, \varphi_{1}\right)=\log 2$.

When we look closer at the system $\left(\Sigma_{2}, \sigma_{2}, \varphi_{0}\right)$, we see that the real reason it should have zero rotational entropy is that there is no rotation in it, that is, the rotation set consists of one point. Thus, we can state our next two conditions as follows.

(7) If the rotation set for $(f, \varphi)$ consists of one point, then $h_{\text {rot }}(f, \varphi)=0$.

(8) $h_{\text {rot }}\left(\sigma_{2}, \varphi_{1}\right)=\log 2$.

It is not clear at first what the rotational entropy should be for more complicated horseshoes. For instance, if we replace the function $\varphi_{1}$ by $3 \varphi_{1}$ (which corresponds to the horseshoe wrapping 3 times around the torus), should the rotational entropy triple or should it remain the same? Condition (5) settles the dispute - the entropy should remain the same. Otherwise, since we deal with the 2-horseshoe, it would exceed the usual topological entropy.

Now, what if we have a horseshoe that is mostly local, but some part of it goes around the torus? Should we count the local part too? Let us look at the next example. Take the full two-sided shift $\sigma_{4}: \Sigma_{4} \rightarrow \Sigma_{4}$ on four symbols $0,1,2,3$, with the function $\varphi$ defined by $\varphi\left(\ldots, x_{-1}, x_{0}, x_{1}, \ldots\right)$ equal to 0 if $x_{0}=0$, to 1 if $x_{0}=1$ or 2 , and to 2 if $x_{0}=3$. Should we have $h_{\text {rot }}\left(\sigma_{4}, \varphi\right)$ equal to $\log 3$ or $\log 4$ ? Since from the point of view of rotation vectors the symbols 1 and 2 are indistinguishable, the answer should be $\log 3$. Alas, this is incompatible with the other postulates we made. The system $\left(\Sigma_{4}, \sigma_{4}, \varphi\right)$ is conjugate to the second iterate of the system $\left(\Sigma_{2}, \sigma_{2}, \varphi_{1}\right)$, so by (8), (2) and (4) we get $h_{\text {rot }}\left(\sigma_{4}, \varphi\right)=\log 4$. 
Since we consider (4) really important, we have to accept the above result. Thus, if only a part of a multiple horseshoe is not local, we count the whole entropy produced by this horseshoe. This will motivate our definitions in the next section.

In fact, the incompatibility of the wishes that we encountered could have been foreseen. It originates in the fact that if we look at the iterates, the size of the set of symbols grows exponentially with the iterate, while the size of the rotation set grows only polynomially.

\section{Definitions}

We use again our standard notation $(X, f, \varphi)$. Although $\varphi$ may have nothing to do with the displacement, we will pretend it is the displacement. Namely, we will refer to a point $x \in X$ moving by $\varphi(x)$ in $\mathbb{R}^{d}$. For instance, if $\varphi\left(f^{i}(x)\right)=v$ for all $i$, we will think of the point $x$ as moving uniformly in the direction of the vector $v$. The rotation vector at $x$ is the limit

$$
\lim _{n \rightarrow \infty} \frac{1}{n} \sum_{i=0}^{n-1} \varphi\left(f^{i}(x)\right)
$$

if this limit exists. If the rotation vector at $x$ exists and is equal to $v$ then $x$ moves in the direction of $v$, but perhaps in a non-uniform way. By the ergodic theorem, for every ergodic invariant measure $\mu$ the rotation vector exists and is equal to $\int \varphi d \mu$ at $\mu$-almost every point (all measures we consider are probability measures, so $\mu(X)=1)$. Sometimes we will refer to this vector as the rotation vector of $\mu$. Note that if $\mu$ and $\nu$ are different ergodic invariant measures then $\mu$-almost every point may mean $\nu$-almost no point and vice versa.

The set of rotation vectors at all points where they exist is called the rotation set of $f$ (or of the pair $(f, \varphi)$ if it is not clear which $\varphi$ we consider).

Our aim is to "partition" topological entropy into parts that come from points moving in concrete directions, in a way that is to some degree uniform, and the part that comes from the rest of the space. To this end we will use ergodic theory. By the variational principle, the topological entropy of $f$ is equal to the supremum of the metric entropies of $f$ for ergodic invariant probability measures on $X$. Thus, we have to classify those measures according to whether they are strongly associated with one direction (and which one) or not. By the support of a measure we will understand the smallest closed subset of $X$ of full measure. If the measure is invariant then obviously its support is also invariant. We will call an ergodic invariant probability measure $\mu$ directional if the rotation set of $f$ restricted to the support of $\mu$ consists of one vector. This vector will be called the direction of $\mu$. An ergodic invariant probability measure that is not directional will be called lost.

Now we define the directional entropy of $f$ in the direction $v \in \mathbb{R}^{d}$ (or briefly, the $v$-entropy of $f$ ) $h_{v}(f, \varphi)$ as the supremum of the metric entropies of $f$ for all directional measures of direction $v$ (when dealing with entropies, we consider the supremum over the empty set to be 0$)$. Similarly, we define the lost entropy $h_{l}(f, \varphi)$ of $f$ as the supremum of the metric entropies of $f$ for all lost measures. Thus, the lost entropy is our version of the rotational entropy. It can be easily checked that it satisfies conditions (1)-(8) from the preceding section.

Since the directional and lost entropies are varieties of topological entropy, one should be able to give more topological definitions (without mentioning measure entropies) of these notions. We will show that they are equal to topological entropies 
of $f$ restricted to some subsets of $X$. Those subsets are not necessarily closed, so we have to use Bowen's definition of topological entropy for noncompact sets from [Bow].

For the directional entropies the situation is simpler than for the lost entropy. We say that a point $x \in X$ is directional of direction $v \in \mathbb{R}^{d}$ if the rotation set of $f$ restricted to the closure of the trajectory of $x$ is $\{v\}$. We denote the set of all directional points of $X$ of direction $v$ by $D(v)$.

To simplify the statement of the next result, we adopt the convention that if $f: \emptyset \rightarrow \emptyset$ then $h(f)=0$. In the proof we will use the notation $V(x)$ for the set of weak* accumulation points of the sequence of measures $\left(\sum_{i=0}^{n-1} \delta_{f^{i}(x)}\right)_{n=1}^{\infty}$. Here $\delta_{x}$ denotes the probability measure concentrated at $x$. The weak* topology is the topology of convergence on all continuous real functions. That is, the measures $\mu_{n}$ weak* converge to $\mu$ if $\int \psi d \mu_{n}$ converges to $\int \psi d \mu$ for every continuous function $\psi: X \rightarrow \mathbb{R}$. The set of all probability measures invariant for $f$ is compact in this topology (see e.g. [DGS]).

Proposition 4.1. For every $v \in \mathbb{R}^{d}$ we have $h_{v}(f, \varphi)=h\left(\left.f\right|_{D(v)}\right)$.

Proof. Take $x \in D(v)$. The closure $Y$ of the trajectory of $x$ is a closed invariant set. Therefore the support of every measure $\mu \in V(x)$ is contained in $Y$. The same is true for almost every ergodic component of $\mu$, so $h_{\mu}(f) \leq h_{v}(f, \varphi)$. Thus, by Theorem 2 of [Bow], $h\left(\left.f\right|_{D(v)}\right) \leq h_{v}(f, \varphi)$.

On the other hand, for every directional measure $\mu$ of direction $v$, the support of $\mu$ is contained in $D(v)$, so $\mu(D(v))=1$. Hence, by Theorem 1 of [Bow], $h_{\mu}(f) \leq$ $h\left(\left.f\right|_{D(v)}\right)$. Thus, $h_{v}(f, \varphi) \leq h\left(\left.f\right|_{D(v)}\right)$.

We can simplify the definition of $h_{v}(f, \varphi)$ even further by finding a simpler characterization of the set $D(v)$. The original definition requires one to look at the time series $\left(\varphi \circ f^{n}\right)_{n=0}^{\infty}$ at all points of the closure of the trajectory of a point $x$ in order to check whether $x \in D(v)$. The following proposition shows that it is enough to look at the time series only at $x$ and allows us to check whether $x \in D(v)$ in a countable number of steps. Moreover, we show that if the rotation set of $f$ restricted to some compact invariant subset of $X$ consists of one vector (as for instance in the definition of a directional measure) then this is the rotation vector at all points of this subset.

Proposition 4.2. The following are equivalent for compact invariant $Y \subset X$ :

(a) The rotation set of $\left.f\right|_{Y}$ is $\{v\}$;

(b) Every $y \in Y$ has rotation vector $v$;

(c) There is a dense subset $Z$ of $Y$ satisfying

$$
\lim _{n \rightarrow \infty}\left(\sup _{y \in Z}\left\|\frac{1}{n} \sum_{i=0}^{n-1} \varphi\left(f^{i}(y)\right)-v\right\|\right)=0 ;
$$

(d) (4.1) is satisfied for $Z=Y$.

In particular, a point $x \in X$ belongs to $D(v)$ if and only if

$$
\lim _{n \rightarrow \infty}\left(\sup _{k}\left\|\frac{1}{n} \sum_{i=k}^{k+n-1} \varphi\left(f^{i}(x)\right)-v\right\|\right)=0 .
$$


Proof. Properties (c) and (d) are equivalent by the continuity of $\varphi$ and $f$. Clearly, (d) implies (b) implies (a). Thus, it remains to prove that (a) implies (d).

Set $b_{n}(y)=(1 / n) \sum_{i=0}^{n-1} \varphi\left(f^{i}(y)\right)$. Suppose that (d) does not hold. Then there is $\varepsilon>0$ and a sequence $\left(y_{j}\right)_{j=1}^{\infty}$ of points from $Y$ such that $\left\|b_{j}\left(y_{j}\right)-v\right\| \geq \varepsilon$ for every $j$. The support of the measure $\mu_{j}=(1 / j) \sum_{i=0}^{n-1} \delta_{f^{i}\left(y_{j}\right)}$ is contained in $Y$. Moreover, $\int \varphi d \mu_{j}=b_{j}\left(x_{j}\right)$. The sequence $\left(\mu_{j}\right)_{j=1}^{\infty}$ has a weak* accumulation point $\mu$. By standard arguments (see e.g. [DGS]) this $\mu$ is an invariant probability measure with support contained in $Y$. Moreover, $\int \varphi d \mu$ is an accumulation point of the sequence $\left(b_{j}\left(x_{j}\right)\right)_{j=1}^{\infty}$, so $\left\|\int \varphi d \mu-v\right\| \geq \varepsilon$. Thus, for some ergodic component $\nu$ of $\mu$ we have also $\left\|\int \varphi d \nu-v\right\| \geq \varepsilon$. Therefore $\int \varphi d \nu \neq v$. Since the support of $\nu$ is also contained in $Y$, the rotation set of $\left.f\right|_{Y}$ cannot be equal to $\{v\}$, so (a) does not hold.

Even if we do not specify the vector, we can still check whether $x$ is directional.

Proposition 4.3. A point $x \in X$ is directional if and only if

$$
\lim _{n \rightarrow \infty}\left(\sup _{k, l}\left\|\frac{1}{n} \sum_{i=k}^{k+n-1} \varphi\left(f^{i}(x)\right)-\frac{1}{n} \sum_{i=l}^{l+n-1} \varphi\left(f^{i}(x)\right)\right\|\right)=0 .
$$

Proof. If $x$ is directional, then (4.2) follows immediately from Proposition 4.2 and the triangle inequality.

Let us assume now that $x$ is not directional. Then there are points $y_{1}, y_{2}$ in the closure of the trajectory of $x$ with rotation vectors $v_{1}, v_{2}$ respectively, and $v_{1} \neq v_{2}$. Set $\varepsilon=(1 / 2)\left\|v_{1}-v_{2}\right\|$. Then there exists $N$ such that for every $n \geq N$

$$
\left\|\frac{1}{n} \sum_{i=0}^{n-1} \varphi\left(f^{i}\left(y_{1}\right)\right)-\frac{1}{n} \sum_{i=0}^{n-1} \varphi\left(f^{i}\left(y_{2}\right)\right)\right\|>\varepsilon .
$$

Since $y_{1}$ and $y_{2}$ can be approximated by points from the trajectory of $x$, we can find $k$ and $l$ (depending on $n$ ) such that

$$
\left\|\frac{1}{n} \sum_{i=0}^{n-1} \varphi\left(f^{i}\left(f^{k}(x)\right)\right)-\frac{1}{n} \sum_{i=0}^{n-1} \varphi\left(f^{i}\left(f^{l}(x)\right)\right)\right\|>\varepsilon .
$$

Therefore (4.2) does not hold.

Finding an invariant set $L$ such that $h\left(\left.f\right|_{L}\right)=h_{l}(f, \varphi)$ and describing the condition $x \in L$ in terms of the time series at $x$ is more difficult. In order to use Theorem 2 of [Bow], we have to have for every $x \in L$ a measure from $V(x)$, almost all of whose ergodic components are lost. As an example from [PP] shows, there is not much chance of replacing a condition of this type with a weaker one.

The simplest thing to do would be to find a characterization of generic points of lost measures by means of time series at those points. However, a simple example shows that this may be impossible.

Let us look at the product of our map $f$ with the 2-shift: $g=f \times \sigma_{2}: X \times \Sigma_{2} \rightarrow$ $X \times \Sigma_{2}$, with the function $\varphi_{g}(x, y)=\varphi(x)$. If $\mu$ is a lost measure for $(f, \varphi)$ and $x$ is a generic point of $\mu$ then for the fixed point $y=(\ldots, 0,0,0, \ldots) \in \Sigma_{2}$ the measure $\mu \times \delta_{y}$ is lost for $\left(g, \varphi_{g}\right)$ and the point $(x, y)$ is generic for it. However, if a point $z \in \Sigma_{2}$ is not generic for any invariant measure (for $\left.\sigma_{2}\right)$ then the point $(x, z)$ is not generic for any invariant measure (for $g$ ). However, the time series at $(x, y)$ and $(x, z)$ are the same. 
Let us analyze the above example further. Let $\nu \in V(x, z)$. Then the projection of $\nu$ onto the first coordinate belongs to $V(x)$, so it is equal to $\mu$. Since $\mu$ is ergodic, almost every ergodic component $\varkappa$ of $\nu$ projects to $\mu$. The projection of the support of $\varkappa$ is a closed invariant subset of the support of $\mu$ and has full $\mu$ measure. Therefore it is equal to the support of $\mu$. Thus, the rotation set of $\left.g\right|_{\operatorname{supp} \varkappa}$ is the same as the rotation set of of $\left.f\right|_{\operatorname{supp} \mu}$, so it consists of more than one point. This proves that $\varkappa$ is lost. Thus, almost every ergodic component of $\nu$ is lost, so the point $(x, z)$ is good for our purposes (that is, it may belong to $L$ ).

This example motivates the following construction. Let us introduce an equivalence relation $\sim$ in $X$, such that $x \sim y$ if and only if the time series at $x$ and $y$ are the same. The equivalence classes of $\sim$ are closed, so we can take the quotient space $\widetilde{X}=X / \sim$. The image of an equivalence class under $f$ is contained in an equivalence class, so we get the induced map $\widetilde{f}: \widetilde{X} \rightarrow \widetilde{X}$. The diagram

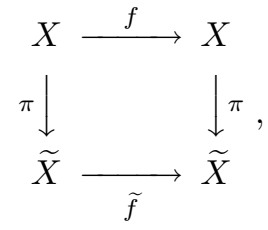

where $\pi$ is the natural projection, commutes. Moreover, since $\varphi$ is constant on each equivalence class, we get a function $\widetilde{\phi}: \widetilde{X} \rightarrow \mathbb{R}^{d}$ such that $\varphi=\widetilde{\phi} \circ \pi$.

Now the new system has the property that the time series at distinct points are distinct. This means that we can use a kind of symbolic dynamics for $\widetilde{f}$. The function $\widetilde{\phi}$ is bounded, so $\widetilde{\phi}(\widetilde{X}) \subset[-M, M]^{d}$ for some $M>0$. Thus, when we apply coding, we get a map $\Psi: \widetilde{X} \rightarrow[-M, M]^{\mathbb{N}}$ defined by taking $\widetilde{\phi}\left(\widetilde{f}^{n}(x)\right)$ as coordinates $(n-1) d$ through $n d-1$ of the point $\Psi(x)$. Then the diagram

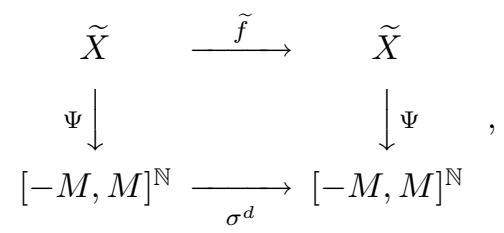

where $\sigma$ is the shift, commutes.

We endow $[-M, M]^{\mathbb{N}}$ with the product topology (so it becomes a Hilbert cube with edge of length $2 M$ ). Since our coding is not quite standard (the space of symbols is uncountable), we have to check how good from the topological point of view it is. It turns out it is very good.

Lemma 4.4. The map $\Psi$ is a homeomorphism onto its image.

Proof. By the definition of $\widetilde{X}$, the map $\Psi$ is one-to-one. The space $\widetilde{X}$ is compact, so it is enough to show that $\Psi$ is continuous. The standard open basis in $[-M, M]^{\mathbb{N}}$ consists of the sets of the form $A=U_{0} \times U_{1} \times \cdots \times U_{n} \times[-M, M]^{\{n+1, n+2, \ldots\}}$, where $U_{i}$ are open subsets of $[-M, M]$. The set $\Psi^{-1}(A)$ is the set of all points of $\widetilde{X}$ satisfying certain conditions of the form $\widetilde{\phi}\left(\widetilde{f}^{j}(x)\right) \in V_{j}$, where $V_{j}$ is open. There are finitely many conditions, so $\Psi^{-1}(A)$ is a finite intersection of open sets, so it is open. This proves that $\Psi$ is continuous.

Now we define $L$ as the set of all points $x \in X$ for which $\pi(x)$ is generic for some lost measure on $\widetilde{X}$. 
We use the following notation. If $g: X \rightarrow Y$ is a continuous map and $\mu$ is a measure on $X$ then $\nu=g_{*}(\mu)$ is the measure on $Y$ given by $\nu(Z)=\mu\left(g^{-1}(Z)\right)$ for Borel subsets $Z \subset Y$. In particular, if $Y=X$ then the condition $g_{*}(\mu)=\mu$ is equivalent to $\mu$ being $g$-invariant.

Proposition 4.5. We have $h_{l}(f, \varphi)=h\left(\left.f\right|_{L}\right)$.

Proof. The proof is similar to what we did in the special case of the space $X \times \Sigma_{2}$ and to the proof of Proposition 4.1.

Let $x \in L$. Then $\pi(x)$ is generic for a lost measure $\mu$ on $\widetilde{X}$. If $\nu \in V(x)$, then $\pi_{*}(\nu)=\mu$. Since $\mu$ is ergodic, almost every ergodic component $\varkappa$ of $\nu$ is such that $\pi_{*}(\varkappa)=\mu$. The set $\pi(\operatorname{supp} \varkappa)$ is a closed invariant subset of $\operatorname{supp} \mu$ and $\mu(\pi(\operatorname{supp} \varkappa))=1$. Therefore $\pi(\operatorname{supp} \varkappa)=\operatorname{supp} \mu$. Thus, the rotation set of $\left.f\right|_{\operatorname{supp} \varkappa}$ is equal to the rotation set of $\left.\widetilde{f}\right|_{\operatorname{supp} \mu}$, so it consists of more than one point. This proves that $\varkappa$ is lost, and hence $h_{\varkappa}(f) \leq h_{l}(f, \varphi)$. This is true for almost every ergodic component of $\nu$, so $h_{\nu}(f) \leq h_{l}(f, \varphi)$. Thus, by Theorem 2 of [Bow], $h\left(\left.f\right|_{L}\right) \leq h_{l}(f, \varphi)$.

On the other hand, if $\nu$ is a lost measure on $X$, then, since $\operatorname{supp} \pi_{*}(\nu)=$ $\pi(\operatorname{supp} \nu)$, the rotation set of $\left.\widetilde{f}\right|_{\operatorname{supp} \pi_{*}(\nu)}$ is equal to the rotation set of $\left.f\right|_{\operatorname{supp} \nu}$, so it consists of more than one point. This means that the measure $\pi_{*}(\nu)$ is lost, and hence $\nu(L)=\pi_{*}(\nu)(\pi(L))=1$. By Theorem 1 of [Bow] we get $h\left(\left.f\right|_{L}\right) \geq h_{\nu}(f)$. This is true for every lost measure $\nu$ on $X$, so $h\left(\left.f\right|_{L}\right) \geq h_{l}(f, \varphi)$.

It remains to show how to recognize from the time series at $x$ whether $x$ belongs to $L$. We cannot of course do this in finitely many steps, so we settle for second best, that is recognition in countably many steps.

Let us denote by $\mathcal{P}$ the set of all functions of the form $P \circ \Phi_{m}: X \rightarrow \mathbb{R}$ for some $m$, where $P$ is a real polynomial in $m d$ real variables with rational coefficients, and $\Phi_{m}: X \rightarrow \mathbb{R}^{m d}$ is given by $\Phi_{m}=\left(\varphi, \varphi \circ f, \ldots, \varphi \circ f^{m-1}\right)$. Note that there are only countably many such functions.

Proposition 4.6. A point $x \in X$ belongs to $L$ if and only if it is not directional and for every $\psi_{1}, \psi_{2} \in \mathcal{P}$

$$
\begin{aligned}
& \lim _{k \rightarrow \infty} \lim _{n \rightarrow \infty}\left(\frac{1}{n} \sum_{i=0}^{n-1} \psi_{1}\left(f^{i}(x)\right) \cdot \frac{1}{k} \sum_{l=0}^{k-1} \psi_{2}\left(f^{i+l}(x)\right)\right) \\
& =\left(\lim _{n \rightarrow \infty} \frac{1}{n} \sum_{i=0}^{n-1} \psi_{1}\left(f^{i}(x)\right)\right) \cdot\left(\lim _{n \rightarrow \infty} \frac{1}{n} \sum_{i=0}^{n-1} \psi_{2}\left(f^{i}(x)\right)\right) .
\end{aligned}
$$

Proof. Assume first that $x \in L$. Then $\pi(x)$ is a generic point for some lost measure $\mu$ on $\widetilde{X}$. Clearly, $x$ is not directional. Since $\varphi \circ f^{i}=\widetilde{\phi} \circ \widetilde{f}^{i} \circ \pi$, we have $\psi_{j}=\widetilde{\psi}_{j} \circ \pi$ for some continuous functions $\psi_{j}$ on $\widetilde{X}(j=1,2)$. Therefore the left-hand side of (4.3) is equal to

$$
\lim _{k \rightarrow \infty} \frac{1}{k} \sum_{l=0}^{k-1} \int \widetilde{\psi}_{1} \cdot\left(\widetilde{\psi}_{2} \circ \widetilde{f}^{l}\right) d \mu,
$$

and the right-hand side of (4.3) is equal to

$$
\int \widetilde{\psi}_{1} d \mu \cdot \int \widetilde{\psi}_{2} d \mu
$$


The functions $\widetilde{\psi}_{j}$ are continuous, so they are integrable, and the measure $\mu$ is ergodic. Thus, (see e.g. [DGS]),

$$
\lim _{k \rightarrow \infty} \frac{1}{k} \sum_{l=0}^{k-1} \int \widetilde{\psi}_{1} \cdot\left(\widetilde{\psi}_{2} \circ \widetilde{f}^{l}\right) d \mu=\int \widetilde{\psi}_{1} d \mu \cdot \int \widetilde{\psi}_{2} d \mu,
$$

so (4.3) holds.

Assume now that $x$ is not directional and (4.3) holds for all $\psi_{1}, \psi_{2} \in \mathcal{P}$. The set $\mathcal{P}$ consists of all functions of the form $P \circ \pi_{m d} \circ \Psi \circ \pi$, where $P$ is a real polynomial in $m d$ variables with rational coefficients and $\pi_{m d}:[-M, M]^{\mathbb{N}} \rightarrow \mathbb{R}^{m d}$ is the projection onto the first $m d$ coordinates. Now, by the Stone-Weierstrass theorem, the set of these polynomials (composed with the corresponding projections) are dense in the space $C\left([-M, M]^{\mathbb{N}}\right)$ of all continuous real functions on $[-M, M]^{\mathbb{N}}$. Thus, since $\Psi$ is a homeomorphism onto its image, the set $\mathcal{R}$ of functions $\widetilde{\psi}$ such that $\widetilde{\psi} \circ \pi \in \mathcal{P}$, is dense in the space $C(\widetilde{X})$. Hence,

$$
\begin{aligned}
& \lim _{k \rightarrow \infty} \lim _{n \rightarrow \infty}\left(\frac{1}{n} \sum_{i=0}^{n-1} \widetilde{\psi}_{1}\left(\widetilde{f}^{i}(\pi(x))\right) \cdot \frac{1}{k} \sum_{l=0}^{k-1} \widetilde{\psi}_{2}\left(\widetilde{f}^{i+l}(\pi(x))\right)\right) \\
= & \left(\lim _{n \rightarrow \infty} \frac{1}{n} \sum_{i=0}^{n-1} \widetilde{\psi}_{1}\left(\widetilde{f}^{i}(\pi(x))\right)\right) \cdot\left(\lim _{n \rightarrow \infty} \frac{1}{n} \sum_{i=0}^{n-1} \widetilde{\psi}_{2}\left(\widetilde{f}^{i}(\pi(x))\right)\right)
\end{aligned}
$$

for every $\widetilde{\psi}_{1}, \widetilde{\psi}_{2} \in C(\widetilde{X})$.

In particular, the $\operatorname{limit}_{n \rightarrow \infty} \frac{1}{n} \sum_{i=0}^{n-1} \widetilde{\psi}_{1}\left(\tilde{f}^{i}(\pi(x))\right)$ exists for every $\widetilde{\psi}_{1} \in C(\tilde{X})$. This limit defines a non-negative linear functional on $C(\widetilde{X})$ of norm 1, that is (by the Riesz Theorem) an invariant probability measure $\mu$ on $\widetilde{X}$, and the point $\pi(x)$ is generic for $\mu$. Now (4.5) tells us that (4.4) holds for all $\widetilde{\psi}_{1}, \widetilde{\psi}_{2} \in C(\widetilde{X})$. The space $C(\widetilde{X})$ is dense in $L_{1}(\mu)$, so (4.4) holds for all $\widetilde{\psi}_{1}, \widetilde{\psi}_{2} \in L_{1}(\mu)$. Therefore $\mu$ is ergodic (see e.g. [DGS]). Since $x$ is not directional, the rotation set of $\left.\widetilde{f}\right|_{\operatorname{supp} \mu}$ consists of more than one point. Therefore $x \in L$.

Note that if we replace our function $\varphi$ by a cohomologous function $\varphi^{\prime}$ then the rotation sets of $f$ restricted to the supports of measures will not change, so the directional and lost entropies will remain the same. By the definition, the sets $D(v)$ will not change, too. However, for $L$ this is not the case.

Let $I$ be the unit interval and let $T: I \rightarrow I$ be the full tent map $(T(x)=1-\mid 2 x-$ $1 \mid$ ). Consider a function $\varphi_{T}: I \rightarrow \mathbb{R}$ that is constant (say, 0 ). Then $\widetilde{I}$ consists of one point. Now look at the function $\varphi_{T}^{\prime}: I \rightarrow \mathbb{R}$ given by $\varphi_{T}^{\prime}(x)=x-T(x)$. With this function, it is easy to check that the only nontrivial pair of equivalent points is the pair of fixed points, $0 \sim 2 / 3$. Thus, in this case $\widetilde{I}$ is homeomorphic to the letter " $\sigma$ ". Nevertheless, $\varphi_{T}$ and $\varphi_{T}^{\prime}$ are cohomologous. Now take a system $\left(X, f, \varphi_{f}\right)$ for which there is a lost measure $\mu$. Form the product $(X \times I, f \times T)$ and define the functions $\varphi, \varphi^{\prime}: X \times I \rightarrow \mathbb{R}^{d+1}$ by $\varphi(x, y)=\left(\varphi_{f}(x), \varphi_{T}(y)\right), \varphi^{\prime}(x, y)=\left(\varphi_{f}(x), \varphi_{T}^{\prime}(y)\right)$. These two functions are cohomologous. However, while for $\varphi$ every point $(x, y)$, where $x$ is generic for $\mu$, belongs to $L$, this is not the case for $\varphi^{\prime}$.

\section{Subshifts of Finite TYPE}

Let $(\Sigma, \sigma)$ be a subshift of finite type. This means that $\Sigma$ is a subset of the space $\{1,2, \ldots, s\}^{\mathbb{N}}$ (or $\{1,2, \ldots, s\}^{\mathbb{Z}}$; we can consider either case) with the product 
topology and $\sigma$ shifts every sequence by one to the left, an $s$ by $s$ 0-1 matrix $M=\left(m_{i j}\right)$ is given, and a sequence $\left(x_{k}\right)$ belongs to $\Sigma$ if and only if $m_{x_{k} x_{k+1}}=1$ for every $k$. Let $G$ be the Markov graph of $(\Sigma, \sigma)$, that is the directed graph whose vertices are $\{1,2, \ldots, s\}$, with an arrow from $i$ to $j$ if and only if $m_{i j}=1$. It is a standard procedure to interpret the elements of $\Sigma$ as infinite paths in $G$.

If we have a function $\varphi: \Sigma \rightarrow \mathbb{R}^{d}$ depending only on the vertices (i.e. constant on cylinders of length 1) then we can speak of ergodic sums and averages for finite paths. In particular, for a loop (a path that ends at the same vertex it begins at) the ergodic sum is the same as the rotation vector of the corresponding periodic point. Hence, we will speak also of rotation vectors of loops (here we think of a loop really as a path, that is a loop has a vertex marked; in other words a loop corresponds to a periodic point, not a periodic orbit). We denote the rotation vector of a loop $L$ by $\varrho(L)$.

We will need the following theorem of Kwapisz ([Kw], Theorem 2.2.4). We restate it slightly so that it fits to our notation.

Theorem 5.1. Let $\mu$ be an invariant ergodic measure on $\Sigma$, let $v=\int \varphi d \mu$, and let $\varepsilon>0$. Then there exists a nonempty compact invariant set $K \subset \Sigma$ and a constant c such that $h\left(\left.\sigma\right|_{K}\right)>h_{\mu}(\sigma)-\varepsilon$ and

$$
\left\|\sum_{i=0}^{n-1} \varphi\left(\sigma^{i}(x)\right)-n v\right\| \leq c
$$

for every $x \in K$ and $n \in \mathbb{N}$.

We get immediate corollaries to this theorem and the variational principle.

Corollary 5.2. If a measure $\mu$ has rotation vector $v$ and $\varepsilon>0$ is given, then there exists a directional measure $\nu$ of direction $v$ with entropy larger than $h_{\mu}(\sigma)-\varepsilon$.

Corollary 5.3. The topological entropy of $\sigma$ is equal to the supremum of the entropies of all directional measures.

Let us investigate what we can get if we try to replace directional measures by lost measures in the above corollaries. The simplest counterexample is when the function $\varphi$ is constant. Then there are no lost measures at all. Other counterexamples can be constructed easily with $v$ being an extreme vector of the rotation set of $\sigma$. However, it turns out that the situation is much better if we consider the interior vectors of the rotation set.

Proposition 5.4. Let $(\Sigma, \sigma)$ be a mixing subshift of finite type, $\varphi: \Sigma \rightarrow \mathbb{R}^{d}(d \geq 1)$ a function depending only on vertices of the Markov graph, and $v$ a vector in the interior of the rotation set $R$ of $(\sigma, \varphi)$. Let $\mu$ be a directional ergodic probability measure, invariant for $\sigma$ and with direction $v$, and let $\varepsilon>0$. Then there exists a lost ergodic probability measure $\nu$, invariant for $\sigma$, with $\int \varphi d \nu=v$ and such that $h_{\nu}(\sigma)>h_{\mu}(\sigma)-\varepsilon$.

Proof. Let $G$ be the Markov graph of $(\Sigma, \sigma)$. Fix a vertex $A$ of $G$. Since $\sigma$ is mixing, there is a constant $m$ such that for every $m^{\prime} \geq m$ and every pair of vertices $B, C$ of $G$ there is a path from $B$ to $C$ of length $m^{\prime}$.

The rotation set $R$ is a polyhedron with vertices given by the rotation vectors of certain loops of $G$ (see e.g. $[\mathrm{Z}]$ ). By repeating such a loop sufficiently many times and adding at the beginning a path from $A$ to the beginning of the loop and at the 
end a path from the end of the loop to $A$ (both paths of length $m$ ) we get loops from $A$ to $A$ with rotation vectors approximating as well as we want the vertices of $R$. Hence, $v$ is in the interior of a simplex with vertices $\varrho\left(L_{0}\right), \ldots, \varrho\left(L_{d}\right)$ for some loops $L_{0}, \ldots, L_{d}$ from $A$ to $A$. Moreover, since repeating a loop does not change the rotation vector, we may assume that the lengths of all the $L_{i}$ are the same (say, $l)$.

In particular, there is $\delta>0$ such that the ball of radius $\delta$ around $v$ is contained in the simplex with vertices $\varrho\left(L_{0}\right), \ldots, \varrho\left(L_{d}\right)$. Therefore, for every $u$ with $\|u-v\| \leq \delta$ there are positive constants $\alpha_{0}, \ldots, \alpha_{d}$ with $\sum \alpha_{i}=1$ such that $\sum \alpha_{i} \varrho\left(L_{i}\right)=u$.

By Theorem 5.1, there exists a nonempty compact invariant set $K \subset \Sigma$ and a constant $c$ such that $h\left(\left.\sigma\right|_{K}\right)>h_{\mu}(\sigma)-\varepsilon / 2$ and

$$
\left\|\sum_{i=0}^{n-1} \varphi\left(\sigma^{i}(x)\right)-n v\right\| \leq c
$$

for every $x \in K$ and $n \in \mathbb{N}$. Let $a_{n}$ be the number of paths of length $n$ represented in $K$. We have

$$
\lim _{n \rightarrow \infty} \frac{1}{n} \log a_{n}=h\left(\left.\sigma\right|_{K}\right)>h_{\mu}(\sigma)-\varepsilon / 2 .
$$

By adjoining appropriate paths at the beginning and end of each of the above $a_{n}$ paths we can get loops from $A$ to $A$ of length $k l$ for some $k$ (the same for all loops). We can do this by fixing the length of the paths that we add at the beginning and the length of the paths that we add at the end. This assures us that the loops we get will be distinct for distinct paths. Moreover the adjoined paths can be taken no longer than $m+l$ each. Therefore we get

$$
k l-n \leq 2(m+l) .
$$

We denote the set of loops we get by $\mathcal{A}$. Recall that the number of elements of $\mathcal{A}$ is $a_{n}$.

Notice that if $n$ is sufficiently large then for any $L \in \mathcal{A}$ and $i \in\{0, \ldots, d\}$ we get $\varrho(L) \neq \varrho\left(L_{i}\right)$, and therefore $L$ is different than any repetition of $L_{i}$. Thus, we get a full shift $\left(\Sigma^{\prime}, \sigma^{k l}\right)$ on $a_{n}+d+1$ symbols, with $\Sigma^{\prime} \subset \Sigma$. The vertices of the Markov graph for $\Sigma^{\prime}$ are the elements of $\mathcal{A}$ and $d+1$ loops of length $k l$ made of $k$ repetitions of loops $L_{0}, \ldots, L_{d}$.

Consider the function $\varphi^{\prime}=(1 / k l) \sum_{i=0}^{k l-1} \varphi \circ \sigma^{i}$ on $\Sigma^{\prime}$. For $x \in \Sigma^{\prime}$ the ergodic averages for $\sigma$ and $\varphi$ and for $\sigma^{k l}$ and $\varphi^{\prime}$ are equal: $(1 / j) \sum_{i=0}^{j-1} \varphi^{\prime}\left(\sigma^{k l i}(x)\right)=$ $(1 / j k l) \sum_{i=0}^{j k l-1} \varphi\left(\sigma^{i}(x)\right)$. Therefore the rotation vectors for $\left(\Sigma^{\prime}, \sigma^{k l}, \varphi^{\prime}\right)$ are the same as for $(\Sigma, \sigma, \varphi)$ (restricted to $\Sigma^{\prime}$ ).

If $L \in \mathcal{A}$ then by (5.1) we get

$$
\|\varrho(L)-v\| \leq \frac{1}{k l}[c+(k l-n) \sup \|\varphi\|] .
$$

Hence, if $w=\left(1 / a_{n}\right) \sum_{L \in \mathcal{A}} \varrho(L)$ then

$$
\|w-v\| \leq \frac{1}{k l}[c+(k l-n) \sup \|\varphi\|] .
$$

By (5.3), the right-hand side of (5.4) tends to 0 as $n$ tends to infinity. Therefore we can find a vector $u$ with $\|u-v\|=\delta$, such that $v=\xi u+(1-\xi) w$, and we will 
have $\xi \rightarrow 0$ as $n \rightarrow \infty$. With $\alpha_{i}$ as at the beginning of the proof, we get

$$
v=\sum_{i=0}^{d} \alpha_{i} \xi \varrho\left(L_{i}\right)+\sum_{L \in \mathcal{A}} \frac{1-\xi}{a_{n}} \varrho(L) .
$$

This means that with the product measure $\varkappa$ on $\Sigma^{\prime}$ obtained by assigning measure $(1-\xi) / a_{n}$ to each element of $\mathcal{A}$ and $\alpha_{i} \xi$ to $L_{i}$ we will get

$$
\int \varphi^{\prime} d \varkappa=v
$$

The system $\left(\Sigma^{\prime}, \sigma^{k l}, \varkappa\right)$ is a Bernoulli shift, so we get

$$
\begin{aligned}
h_{\varkappa}\left(\sigma^{k l}\right) & =-\sum_{L \in \mathcal{A}} \frac{1-\xi}{a_{n}} \log \frac{1-\xi}{a_{n}}-\sum_{i=0}^{d} \alpha_{i} \xi \log \left(\alpha_{i} \xi\right) \geq-\frac{1-\xi}{a_{n}} a_{n} \log \frac{1-\xi}{a_{n}} \\
& =(1-\xi) \log a_{n}-(1-\xi) \log (1-\xi) \geq(1-\xi) \log a_{n} .
\end{aligned}
$$

Now we consider the measure $\nu=(1 / k l) \sum_{i=0}^{k l-1} \sigma_{*}^{i}(\varkappa)$. Since $\sigma_{*}^{k l}(\varkappa)=\varkappa$, we get $\sigma_{*}(\nu)=\nu$, so $\nu$ is invariant with respect to $\sigma$. Clearly $\nu(\Sigma)=1$. To see that $\nu$ is ergodic, assume that $M$ is a $\sigma$-invariant subset of $\Sigma$. Then it is also $\sigma^{k l}$-invariant, so $\varkappa(M)=0$ or 1 . Since $M$ is $\sigma$-invariant, $\sigma_{*}^{i}(\varkappa)(M)=\varkappa(M)$, so $\nu(M)$ is also 0 or 1.

We have

$$
\begin{aligned}
\int \varphi^{\prime} d \varkappa & =\int\left(\frac{1}{k l} \sum_{i=0}^{k l-1} \varphi \circ \sigma^{i}\right) d \varkappa=\frac{1}{k l} \sum_{i=0}^{k l-1} \int\left(\varphi \circ \sigma^{i}\right) d \varkappa \\
& =\frac{1}{k l} \sum_{i=0}^{k l-1} \int \varphi d \sigma_{*}^{i}(\varkappa)=\int \varphi d \mu
\end{aligned}
$$

so by (5.5) we get $\int \varphi d \mu=v$.

Since $\left(\Sigma, \sigma^{k l}, \sigma_{*}^{i}(\varkappa)\right)$ is a factor of $\left(\Sigma, \sigma^{k l}, \varkappa\right)$, and $\left(\Sigma, \sigma^{k l}, \varkappa\right)=\left(\Sigma, \sigma^{k l}, \sigma_{*}^{k l}(\varkappa)\right)$ is a factor of $\left(\Sigma, \sigma^{k l}, \sigma_{*}^{i}(\varkappa)\right)$, we have $h_{\sigma_{*}^{i}(\varkappa)}\left(\sigma^{k l}\right)=h_{\varkappa}\left(\sigma^{k l}\right)$. Therefore (see e.g. [DGS]) $h_{\nu}\left(\sigma^{k l}\right)=h_{\varkappa}\left(\sigma^{k l}\right)$. Hence $h_{\nu}(\sigma)=(1 / k l) h_{\varkappa}\left(\sigma^{k l}\right)$, so by $(5.6)$,

$$
h_{\nu}(\sigma) \geq \frac{1-\xi}{k l} \log a_{n}
$$

By (5.3), $k l / n \rightarrow 1$ as $n \rightarrow \infty$, and we know that $\xi \rightarrow 0$ as $n \rightarrow \infty$. Hence, the right-hand side of (5.7) tends to $(1 / n) \log a_{n}$ as $n \rightarrow \infty$. Thus, by (5.2), if $n$ is sufficiently large then $h_{\nu}(\sigma)>h_{\mu}(\sigma)-\varepsilon$.

For $\nu$-almost every $x \in \Sigma$ there are arbitrarily long repetitions of the loop $L_{0}$ in the path representing $x$. Since $\varrho\left(L_{0}\right) \neq v$, this proves that the measure $\nu$ is not directional of direction $v$. Since $\int \varphi d \nu=v$, the measure $\nu$ is not directional, i.e. it is lost.

Let us think how we can weaken the assumptions of Proposition 5.4. First, instead of assuming that $\sigma$ is mixing, it is enough to assume that it is transitive. On the level of the Markov graph this means that there is a path from every vertex to every vertex. If $\sigma$ is transitive but not mixing, then there is $r>1$ and a partition of $\Sigma$ into $r$ sets, each mapped onto the next cyclically, such that $\sigma^{r}$ on each of them is a mixing subshift of finite type (we take new vertices of the Markov graph corresponding to old paths of length $r$ ). These systems are conjugate to one another (including the new observable, which is equal to the ergodic sum of the old 
one over a path of length $r$ ). Therefore they have the same rotation sets, which are equal to the old rotation set multiplied by $r$. Moreover, there is a one-to-one correspondence between the ergodic invariant measures of each of the new systems and of the old system. Therefore application of Proposition 5.4 to any of the new systems proves this proposition with the assumption of transitivity of $\sigma$.

Next, we do not have to assume that $\mu$ is directional, since we can start by applying Corollary 5.2.

Finally, let us think about the assumption of $v$ being in the interior of $R$. To get rid almost completely of this assumption we use the scaffold technique (see [MT], $[\mathrm{Kw}])$. First, instead of the interior of $R$ we can think of the relative interior of $R$, that is its interior relative to the smallest affine subspace containing $R$. This clearly does not change anything in the proof (alternately, we can compose $\varphi$ with the projection onto this affine subspace and then with the isometry with the corresponding Euclidean space). Of course, we assumed that the dimension $d$ of the target space $\mathbb{R}^{d}$ of $\varphi$ is positive, so here we have to assume that this affine subspace is of positive dimension.

Now we need some auxiliary results. We consider a transitive Markov graph $G$ and an observable $\varphi$ constant on 1-cylinders, with rotation set $R$.

Lemma 5.5. Assume that $\varphi$ is real-valued and that $R=[0,1]$. Let $\nu$ be an ergodic measure with rotation number 0 . Then there is an arrow in $G$ (a 2-cylinder) of measure 0.

Proof. By [Z], there is a loop $L$ in $G$ with rotation number 1. Assume that every arrow in this loop has positive measure. Take such an arrow $A=(I \rightarrow J)$. In a $\nu$-generic path $Q$ this arrow appears with positive density. Hence, there is a number $k$ such that the density of blocks of length $k$ with two or more arrows $A$ is positive. Since there are finitely many blocks of length $k$, there is one that contains at least two arrows $A$ and appears in $Q$ with positive density. Thus, there is a finite path $P(A)=J \rightarrow \cdots \rightarrow I$ which appears following $I$ in $Q$ with positive density $p$. This path $P(A)$ is equal to a loop $K$ containing the arrow $I \rightarrow J$, cut between $I$ and $J$. Thus the contribution per vertex of $P(A)$ to the rotation number is the same as the rotation number of $K$, which is a number $c \in[0,1]$. If we cut out all copies of $P(A)$ following $I$ in $Q$, we still get some path $Q^{\prime}$ in $G$. Since the rotation number of $Q$ is 0 , we get that the rotation number of $Q^{\prime}$ is $-p c /(1-p)$. This number must be nonnegative, so we get $c=0$.

If the consecutive arrows of $L$ are $A_{1}, \ldots, A_{s}$ then we can form a loop $M$ by concatenating the paths $P\left(A_{s}\right), \ldots, P\left(A_{1}\right)$. The sum of $\varphi$ along $M$ is equal to the sum over $i=1, \ldots, s$ of sums of the values of $\varphi$ along the paths $P\left(A_{i}\right)$ minus the sum of $\varphi$ along $L$ (each vertex of $L$ is the beginning of some $P\left(A_{i}\right)$ and the end of $\left.P\left(A_{i+1}\right)\right)$. The average value of $\varphi$ along each $P\left(A_{i}\right)$ is 0 and along $L$ is 1 . Thus the average value of $\varphi$ along $M$ (that is, the rotation number of $M$ ) is negative, a contradiction. Hence, there must be an arrow in $L$ with measure zero.

Corollary 5.6. If $\nu$ is an ergodic measure with rotation vector on the relative boundary of $R$ and $R$ consists of more than one point then there is an arrow in $G$ of measure 0 .

A loop in $G$ is called elementary if it is not a concatenation of two shorter loops (that is, it does not pass more than once through any vertex of $G$ ). By [Z], $R$ is a polyhedron (sometimes a polyhedron of an arbitrary dimension is called a polytope) 
and its vertices are rotation vectors of elementary loops. However, there may exist elementary loops whose rotation vectors are not vertices of $R$. We define a subvertex as a rotation vector of an elementary loop that lies on the relative boundary of the rotation set. In particular, all vertices of $R$ are also subvertices. Of course there are only finitely many subvertices for a given $G$ and $\varphi$.

Thus, we get the following final result. We state it for simplicity for transitive systems; otherwise the system decomposes into a finite number of transitive ones and we can apply the theorem to each of them.

Theorem 5.7. Let $(\Sigma, \sigma)$ be a transitive subshift of finite type, $\varphi: \Sigma \rightarrow \mathbb{R}^{d}(d \geq 1)$ a function depending only on vertices of the Markov graph, and $v$ a vector of the rotation set of $(\sigma, \varphi)$ which is not a subvertex. Let $\mu$ be an ergodic invariant measure with $\int \varphi d \mu=v$, and let $\varepsilon>0$. Then there exists a lost measure $\nu$, with $\int \varphi d \nu=v$ and such that $h_{\nu}(\sigma)>h_{\mu}(\sigma)-\varepsilon$.

Proof. If $v$ is in the relative interior of the rotation set, we just use Proposition 5.4 and the discussion following it. If it is on the relative boundary, we remove all arrows and vertices of measure zero from $G$ and we get a new graph $G^{\prime}$. Since $\mu$ is ergodic, $G^{\prime}$ is transitive. Since $v$ was not a subvertex, the rotation set of the new system consists of more than one point. By Corollary $5.6 v$ belongs to its relative interior and again we can use Proposition 5.4.

Corollary 5.8. The topological entropy of $\sigma$ is equal to the maximum of the lost entropy and the directional entropies in the directions of the subvertices of the rotation set.

\section{THE CIRCLE}

In this section we assume that $X$ is the circle (the unit circle in the complex plane), $f: X \rightarrow X$ is a continuous map of degree one, and $\varphi: X \rightarrow \mathbb{R}$ is the displacement function. This means that if $F: \mathbb{R} \rightarrow \mathbb{R}$ is a lifting of $f$, then $\varphi(e(x))=F(x)-x$ for $x \in \mathbb{R}$ (here $e: \mathbb{R} \rightarrow X$ is the natural projection $e(x)=$ $\exp (2 \pi i x))$.

We know how the directional and lost entropies behave on horseshoes. For circle maps a horseshoe is a finite family of closed arcs $J_{1}, \ldots, J_{s}$ with pairwise disjoint interiors such that each arc $f$-covers each arc (we say that an arc $I f$-covers an arc $J$ if there is a subarc $I^{\prime}$ of $I$ such that $f\left(I^{\prime}\right)=J$ ). A horseshoe consisting of $s$ arcs is called an $s$-horseshoe.

It is known (see [MS], [M], [ALM]) that the topological entropy of $f$ is due to horseshoes. More precisely, if $h(f)>0$, then there exist sequences $\left(k_{n}\right)_{n=1}^{\infty}$ and $\left(s_{n}\right)_{n=1}^{\infty}$ of positive integers such that for each $n$ the map $f$ has an $s_{n}$-horseshoe and

$$
\lim _{n \rightarrow \infty} \frac{1}{k_{n}} \log s_{n}=h(f) .
$$

It is natural to ask whether similar results hold for the directional and lost entropies with the corresponding kinds of horseshoes. This requires precise definitions of directional (local) and lost horseshoes. This is easier to do if we modify slightly our definition of a horseshoe.

Statements of some lemmata will become much clearer if we require that the $\operatorname{arcs} J_{i}$ are pairwise disjoint. With this condition we speak of a strong horseshoe (see e.g. $[\mathrm{LM}]$ ). Since existence of an $s$-horseshoe for $f^{n}$ implies existence of an 
$s^{m}$-horseshoe for $f^{n m}$ and this implies existence of an $\left(s^{m}-2\right)$-strong horseshoe for $f^{n m}$ (see $[\mathrm{LM}]$ ), in the theorem from the preceding paragraph we can replace horseshoes by strong horseshoes.

Let $H=\left\{J_{1}, \ldots, J_{s}\right\}$ be a strong horseshoe. By the definition, for every $i, j \in$ $\{1, \ldots, s\}$ there exists a subarc $J_{i}^{j}$ of $J_{i}$ such that $f\left(J_{i}^{j}\right)=J_{j}$. We will call the pair $W=(H, B)$, where $B=\left\{J_{i}^{j}\right\}_{i, j=1}^{s}$ a horseboot, or if we want to specify $s$, an $s$-horseboot. Further, we denote by $K(W)$ the set of those points whose whole trajectory is contained in the union of the $\operatorname{arcs} J_{i}^{j}$. This set is clearly closed and invariant. If the rotation set of $\left.f\right|_{K(W)}$ consists of one point $v \in \mathbb{R}$ then we will call the horseboot $W$ directional of direction $v$. If it consists of more than one point, we will call $W$ lost.

We will also use the terms "horseshoe", "strong horseshoe" and "horseboot" for analogous objects for maps of the real line into itself.

For future reference, let us state the theorem on horseshoes in our newly defined terms.

Theorem 6.1. Let $f: X \rightarrow X$ be a continuous circle map of degree 1 . If $h(f)>0$ then there exist sequences $\left(k_{n}\right)_{n=1}^{\infty}$ and $\left(s_{n}\right)_{n=1}^{\infty}$ of positive integers such that for each $n$ the map $f$ has an $s_{n}$-horseboot and (6.1) holds.

If the rotation set of $f$ consists of one point $v$, then the situation is clear - all invariant ergodic probability measures on $X$ are directional in direction $v$. Therefore the lost entropy and directional entropies in directions other than $v$ are zero, while directional entropy in direction $v$ is equal to the topological entropy of $f$. Moreover, all horseboots are directional in direction $v$, so if $h(f)$ is positive, there exist sequences $\left(k_{n}\right)_{n=1}^{\infty}$ and $\left(s_{n}\right)_{n=1}^{\infty}$ of positive integers such that for each $n$ the map $f$ has an $s_{n}$-horseboot and (6.1) holds. Note that this implies existence of periodic points, so $v$ is rational. Therefore for an irrational $v$ the topological entropy of $f$ is zero.

This means that we have to concentrate on the case when the rotation set of $f$ consists of more than one point. We will work under this assumption until the end of the proof of Lemma 6.8. The rotation set is a closed interval $L$ and for every $v \in L$ there is a point with rotation number $v$ (see e.g. [ALM]).

First we consider the lost entropy and lost horseboots. It turns out that we can apply a factorization that is stronger than the one from Section 4. Namely, we define a reflexive relation $\simeq$ as follows. Distinct points $x, y \in X$ are equivalent if there exists a closed arc $J$ with endpoints $x$ and $y$ such that $f^{n}(J) \neq X$ for every $n$.

Lemma 6.2. The relation $\simeq$ is an equivalence relation.

Proof. The only property requiring proof is transitivity of $\simeq$. Let us take distinct $x, y, z \in X$ and assume that $x \simeq y$ and $y \simeq z$. We want to prove that $x \simeq z$. There exist closed $\operatorname{arcs} I, J$ with endpoints $x, y$ and $y, z$ respectively such that $f^{n}(I) \neq X$ and $f^{n}(J) \neq X$ for every $n$. The cases $I \subset J$ and $J \subset I$ are trivial, so we assume that those inclusions do not hold. Then $I \cup J$ either is an arc with endpoints $x, z$, or is equal to $X$. Suppose that $x$ and $z$ are not equivalent. Then there is $n$ such that $f^{n}(I \cup J)=X$. By our assumptions on the rotation set of $f$ there exist three points $t_{1}, t_{2}, t_{3} \in X$ with three different rotation numbers. Hence, in one of the sets $f^{n}(I), f^{n}(J)$ there are two points $s_{1}, s_{2}$ with different rotation numbers. One of the closed arcs with endpoints $s_{1}, s_{2}$ is contained in $f^{n}(I)$ or $f^{n}(J)$. Call this arc 
$K$. In the lifting, the lengths of the images of a component of $e^{-1}(K)$ converge to infinity, so for some $m$ this length is larger than 1 . This means that $f^{m}(K)=X$. Thus, either $f^{n+m}(I)=X$ or $f^{n+m}(J)=X$, a contradiction. Thus, $x \simeq z$.

Lemma 6.3. Any equivalence class of $\simeq$ either consists of one point or is a closed arc.

Proof. Assume that $A$ is an equivalence class of $\simeq$ that consists of more than one point. By the definition of $\simeq$, there cannot be two points of $A$ with different rotation numbers. Since we assumed that the rotation set of $f$ consists of more than one point, there exist three points of $X$ with three distinct rotation numbers. At least two of those three points do not belong to $A$. Again by the definition of $\simeq$, if $x, y \in A$ and $x \neq y$, then one of the arcs joining $x$ and $y$ is contained in $A$. Therefore $A$ is connected, so it is an arc with different endpoints (so for instance the situation when $A=X \backslash\{y\}$ for some $y \in X$ is excluded). It remains to prove that it is closed.

The closure $J$ of $A$ is a closed arc. For any $n$ the set $f^{n}(A)$ is a connected set contained in some equivalence class. This equivalence class is, by the preceding argument, an arc with distinct endpoints, so $f^{n}(A)$ is also an arc with different endpoints. Then the closure of $f^{n}(A)$ is a closed arc, and it is equal to $f^{n}(J)$. This shows that each two points of $J$ are equivalent. Therefore $J=A$, so $A$ is closed.

The quotient space $X / \simeq$ is also the circle (that is, it is homeomorphic to the circle, but since at this moment only topology is important, we may assume it is also $X$ ). We will denote this quotient map by $\pi: X \rightarrow X$. Equivalence classes are invariant, so we get a map of the quotient $\widehat{f}: X \rightarrow X$. All this can be done also on the level of liftings, so if $\widehat{F}: \mathbb{R} \rightarrow \mathbb{R}$ is a lifting of $\widehat{f}$ and $\Pi: \mathbb{R} \rightarrow \mathbb{R}$ is the quotient map for liftings, then the integer parts of $F(t)-t$ and $\widehat{F}(\Pi(t))-\Pi(t)$ are the same. Therefore when we use the usual displacement function $\varphi: X \rightarrow \mathbb{R}$ for both $f$ and $\widehat{f}$, the rotation numbers at $x$ and $\pi(x)$ exist simultaneously and are equal (if they exist). In particular, the rotation sets of $f$ and $\widehat{f}$ are equal.

The map $\widehat{f}$ is leo (locally eventually onto). That is, if $U$ is an open nonempty subset of $X$ then there is $n$ such that $\widehat{f}^{n}(U)=X$.

Lemma 6.4. Let $\mu$ be an ergodic $f$-invariant probability measure on $X$ and let $\nu=\pi_{*}(\mu)$. If one of the measures $\mu, \nu$ is lost then the other one is also lost and the systems $(X, f, \mu)$ and $(X, \widehat{f}, \nu)$ are isomorphic (in the sense of isomorphism of measure preserving transformations).

Proof. Since $\mu$ is ergodic, so is $\nu$. Therefore $\nu$ either is concentrated on a periodic orbit or is nonatomic. If it is concentrated on a periodic orbit $P$, then $\operatorname{supp} \mu \subset$ $\pi^{-1}(P)$. The rotation set of $\left.f\right|_{\pi^{-1}(P)}$ is the same as the rotation set of $\left.\widehat{f}\right|_{P}$, which consists of one point. Therefore in this case neither $\mu$ nor $\nu$ is lost.

Consider now the case of nonatomic $\nu$. Let $A \subset X$ be the union of those equivalence classes of $\simeq$ that are arcs. There are at most countably many such arcs, so the set $\pi(A)$ is at most countable. Therefore $\mu(A)=\nu(\pi(A))=0$. On the set $X \backslash A$ the map $\pi$ is one to one, and thus the systems $(X, f, \mu)$ and $(X, \widehat{f}, \nu)$ are isomorphic.

The support of $\mu$ is contained in $\pi^{-1}(\operatorname{supp} \nu)$. On the other hand, if $x \in \operatorname{supp} \nu$, then there is a point of $\operatorname{supp} \mu$ in $\pi^{-1}(x)$. Therefore the rotation sets of $\left.f\right|_{\operatorname{supp} \mu}$ 
and $\left.\widehat{f}\right|_{\text {supp } \nu}$ are equal. This proves that either both $\mu, \nu$ are lost, or both are directional.

Lemma 6.5. The lost entropies of $f$ and $\widehat{f}$ are equal.

Proof. By Lemma 6.4, if $\mu$ is a lost measure for $f$ then $\pi_{*}(\mu)$ is a lost measure for $\widehat{f}$ and the entropies of $\mu$ and $\nu$ are equal. This proves that $h_{l}(\widehat{f}, \varphi) \geq h_{l}(f, \varphi)$.

Conversely, let $\nu$ be a lost measure for $\widehat{f}$. There exists a probability measure $\varkappa$ invariant for $f$ such that $\pi_{*}(\varkappa)=\nu$. Almost every ergodic component $\mu$ of $\varkappa$ is such that $\pi_{*}(\mu)=\nu$. By Lemma $6.3, \mu$ is lost and the entropies of $\mu$ and $\nu$ are equal. This proves that $h_{l}(\widehat{f}, \varphi) \leq h_{l}(f, \varphi)$.

Now let us investigate lost horseboots. We have to prove first that they contribute to the lost entropy.

Lemma 6.6. Assume that $f^{n}$ has a horseboot $W=\left(\left\{J_{i}\right\}_{i=1}^{s},\left\{J_{i}^{j}\right\}_{i, j=1}^{s}\right)$. Let $\Sigma_{s}$ be the space of one-sided sequences of elements of $\{1, \ldots, s\}$ with the shift $\sigma$. Then there exists a continuous map $\eta$ of $K(W)$ onto $\Sigma_{s}$ such that the diagram

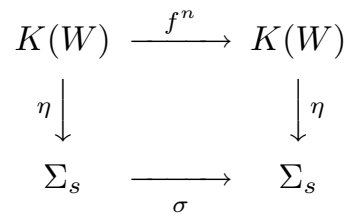

commutes and for every $i, j$ the image $\eta\left(J_{i}^{j}\right)$ is the cylinder $C_{i, j}=\left\{\left(r_{k}\right)_{k=0}^{\infty} \in\right.$ $\left.\Sigma_{s}: r_{0}=i, r_{1}=j\right\}$. Moreover, there exists an integer-valued function $\varphi_{\sigma}$ on $\Sigma_{2}$, constant on cylinders $C_{i, j}$, such that on $K(W)$ the functions $\varphi_{\sigma} \circ \eta$ and $\varphi_{f_{n}}$ are cohomologous (here $\varphi_{f^{n}}$ is the natural displacement for $f^{n}$ ).

Proof. We will use symbolic dynamics. Since the horseshoe $\left\{J_{i}\right\}_{i=1}^{s}$ is strong, for every $x \in K(W)$ there exists a unique sequence $\eta(x)=\left(i_{m}\right)_{m=0}^{\infty}$ such that $f^{n m}(x) \in$ $J_{i_{m}}$ for every $m$. This defines a function $\eta: K(W) \rightarrow \Sigma_{s}$. Clearly, the diagram (6.2) commutes. By the standard arguments, $\eta$ is continuous. Moreover, by the definition of $K(W)$ we have

$$
f^{n m}(x) \in J_{i_{m}}^{i_{m+1}}
$$

for every $m$. Clearly, if (6.3) holds then $x \in K(W)$ and $\eta(x)=\left(i_{m}\right)_{m=0}^{\infty}$.

We claim that $\eta(K(W))=\Sigma_{s}$. To prove the claim, we construct by induction a descending sequence of closed $\operatorname{arcs} I_{k}$ such that for each $x \in I_{k}$ (6.3) holds for $i=0, \ldots, k-1$ and $f^{n k}\left(I_{k}\right)=J_{i_{k}}$. To start the induction we set $I_{1}=J_{i_{0}}^{i_{1}}$. To make the induction step from $k$ to $k+1$, we note that since $f^{n k}\left(I_{k}\right)=J_{i_{k}}$ and $J_{i_{k}}^{i_{k+1}} \subset J_{i_{k}}$, there exists a closed arc $I_{k+1} \subset I_{k}$ such that $f^{n k}\left(I_{k+1}\right)=J_{i_{k}}^{i_{k+1}}$, and this arc satisfies our conditions. Now, the intersection $I=\bigcap_{k=1}^{\infty} I_{k}$ is nonempty and any $x \in I$ satisfies (6.3) for any $m$. This proves the claim.

Let us define a displacement function $\varphi_{\sigma}$ on $\Sigma_{s}$. For each $i$ we choose one connected component $M_{i}$ of $e^{-1}\left(J_{i}\right)$ and then set $M_{i}^{j}=M_{i} \cap e^{-1}\left(J_{i}^{j}\right)$. For any $i, j \in\{1, \ldots, s\}$ there exists an integer $m_{i}^{j}$ such that if $t \in M_{i}^{j}$ then $F^{n}(t)-m_{i}^{j} \in M_{j}$. We set $\varphi_{\sigma}\left(\left(i_{k}\right)_{k=0}^{\infty}\right)=m_{i_{0}}^{i_{1}}$.

If $x \in K(W) \cap J_{i}$ then the set $M_{i} \cap e^{-1}(x)$ consists of one point, and we denote this point by $\zeta(x)$. This defines a bounded Borel (even continuous) function $\zeta$ : 
$K(W) \rightarrow \mathbb{R}$. If $x \in K(W) \cap M_{i}^{j}$ then $\zeta\left(f^{n}(x)\right)=F^{n}(\zeta(x))-m_{i}^{j}$, so $\varphi_{\sigma}(\eta(x))=$ $m_{i}^{j}=F^{n}(\zeta(x))-\zeta\left(f^{n}(x)\right)=\left[F^{n}(\zeta(x))-\zeta(x)\right]+\left[\zeta(x)-\zeta\left(f^{n}(x)\right)\right]=\varphi_{f^{n}}(x)+\zeta(x)-$ $\zeta(f(x))$. Hence, on $K(W)$ the functions $\varphi_{\sigma} \circ \eta$ and $\varphi_{f^{n}}$ are cohomologous.

Lemma 6.7. If $f^{n}$ has a lost $s$-horseboot then $h_{l}(f, \varphi) \geq(1 / n) \log s$.

Proof. We assume that $f^{n}$ has a lost horseboot $W$ and use the notation from Lemma 6.6. Since $\varphi_{\sigma} \circ \eta$ and $\varphi_{f^{n}}$ are cohomologous on $K(W)$, the map $\eta$ preserves rotation numbers. In particular, the rotation set of $\sigma$ is the same as the rotation set of $\left.f\right|_{K(W)}$, so it consists of more than one point.

Let $\nu$ be the Bernoulli $(1 / s, \ldots, 1 / s)$ measure on $\Sigma_{s}$. There exists a probability measure $\varkappa$ invariant for $f^{n}$ such that $\eta_{*}(\varkappa)=\nu$. Since $\nu$ is ergodic, almost every ergodic component $\mu$ of $\varkappa$ is such that $\eta_{*}(\mu)=\nu$. Choose one such $\mu$. The set $\eta(\operatorname{supp} \mu)$ is closed and has full measure $\nu$, so it is equal to the whole $\Sigma_{s}$. Hence the rotation set of $\left.f^{n}\right|_{\operatorname{supp} \mu}$ is the same as the rotation set of $\sigma$, so it consists of more than one point. This shows that the measure $\mu$ is lost. Since $\left(\Sigma_{s}, \sigma, \nu\right)$ is a factor of $\left(K(W),\left.f^{n}\right|_{K(W)}, \mu\right)$, we have $h_{\mu}\left(f^{n}\right) \geq h_{\nu}(\sigma)=\log s$ (we can write $h_{\mu}\left(f^{n}\right)$ instead of $h_{\mu}\left(\left.f^{n}\right|_{K(W)}\right)$, since the systems $\left(K(W),\left.f^{n}\right|_{K(W)}, \mu\right)$ and $\left(X, f^{n}, \mu\right)$ are isomorphic). Therefore $h_{l}(f, \varphi)=(1 / n) h_{l}\left(f^{n}, \varphi_{f^{n}}\right) \geq(1 / n) \log s$.

Now we show that for $\widehat{f}$ it is easy to lose a horseboot.

Lemma 6.8. If $\widehat{f}^{n}$ has an s-horseboot then there exist $n^{\prime}$ and $s^{\prime}$ such that $\widehat{f}^{n^{\prime}}$ has a lost $s^{\prime}$-horseboot and $\left(1 / n^{\prime}\right) \log s^{\prime} \geq(1 / n) \log s$.

Proof. Assume that $\widehat{f}^{n}$ has an $s$-horseboot $W=\left(\left\{J_{i}\right\}_{i=1}^{s},\left\{J_{i}^{j}\right\}_{i, j=1}^{s}\right)$. If it is lost, we are done. Therefore assume that it is directional.

By Lemma 6.6, there is a semiconjugacy $\eta$ between $\left(K(W),\left.\widehat{f}\right|_{K(W)}\right)$ and the onesided $s$-shift. Moreover, there is an integer-valued function $\varphi_{\sigma}$ on $\Sigma_{2}$, constant on cylinders $C_{i, j}$, such that on $K(W)$ the functions $\varphi_{\sigma} \circ \eta$ and $\varphi_{\widehat{f}^{n}}$ are cohomologous.

This function is defined as in the proof of Lemma 6.6 (with $f$ replaced by $\widehat{f}$ ). Since $W$ is directional, the rotation set for $\sigma$ consists of one point. This means that the rotation number for all periodic points of $\sigma$ is the same. This includes fixed points, so this rotation number is an integer. By switching to a different lifting $G$ of $\widehat{f}^{n}$, we can make this number 0 . Moreover, if $x \in C_{i, j}$ and $\sigma_{m}(x) \in C_{i^{\prime}, j^{\prime}}$ then the sum of the values of $\varphi_{\sigma}$ on this piece of the trajectory of $x$ depends only on $i, j, i^{\prime}, j^{\prime}$, not on $x, m$. In terms of the lifting $G$ this means that if $t \in M_{i}^{j} \cap e^{-1}(K(W))$ and $G^{m}(t) \in e^{-1}\left(J_{i^{\prime}}^{j^{\prime}}\right)$, then it does not depend on $t$ and $m$ in which component of $e^{-1}\left(J_{i^{\prime}}^{j^{\prime}}\right)$ the point $G^{m}(t)$ is. Therefore if $N$ is the union over all $i, j$ of the components $N_{i}^{j}$ of $e^{-1}\left(J_{i}^{j}\right)$ to which we can get from $M_{0}^{0} \cap e^{-1}(K(W))$, then $e$ conjugates $G$ on the set $N \cap e^{-1}(K(W))$ with $\widehat{f}^{n}$ on the set $K(W)$.

We have $\widehat{f}^{n}\left(J_{i}^{j}\right)=J_{j}$. Therefore $G\left(N_{i}^{j}\right)$ is a component of the set $e^{-1}\left(J_{j}\right)$. Moreover, it contains the intervals $N_{j}^{k}$ for all $k$. This property determines this component, so it is the same component for all $i$. We call it $N_{j}$. Thus, $N_{j}^{k} \subset N_{j}$ for all $k$. This means that $\left(\left\{N_{i}\right\}_{i=1}^{s},\left\{N_{i}^{j}\right\}_{i, j=1}^{s}\right)$ is a horseboot for $G$. In particular, $\left\{N_{i}\right\}_{i=1}^{s}$ is a strong horseshoe for $G$. By the standard argument (see e.g. [ALM]; in fact this is a construction like the one from the proof of our Lemma 6.6) we see that $G^{m}$ has a strong $s^{m}$-horseshoe $\left\{S_{i}\right\}_{i=1}^{s^{m}}$ with $\bigcup_{i=1}^{s^{m}} S_{i} \subset \bigcup_{j=1}^{s} N_{j}$ and the convex hulls of $\bigcup_{i=1}^{s^{m}} S_{i}$ and $\bigcup_{j=1}^{s} N_{j}$ equal to the same interval $S$. There exists 
a closed interval $I \subset S$ such that $e(I)$ is disjoint from $e\left(\bigcup_{j=1}^{s} N_{j}\right)$. Since $\widehat{f}$ is leo and its rotation set consists of more than one point, there are two points in $I$ with different rotation numbers. Therefore the lengths of $G^{m}(I)$ tend to infinity. Fix $m$ so large that the length of $G^{m}(I)$ is larger than the length of $S$ plus 3.

For every $i$ the set $G^{m}\left(S_{i}\right)$ is an interval containing all $S_{i}$. Thus, it contains $S$, and since $I \subset S$, it contains $I$. On the other hand, $G^{m}(I)$ contains $S$ translated by some integer. Therefore $H=\left\{e\left(S_{i}\right)\right\}_{i=1}^{s^{m}} \cup\{e(I)\}$ is a strong horseshoe for $\widehat{f}^{n m}$. Therefore there exists a collection of $\operatorname{arcs} B$ such that $W^{\prime}=(H, B)$ is a horseboot. We can choose $B$ in such a way that the intervals not involving $e(I)$ are projections to the circle of the elements of some collection of intervals $B^{\prime}$ such that $\left(\left\{S_{i}\right\}_{i=1}^{s^{m}}, B^{\prime}\right)$ is a horseboot for $G^{m}$. Moreover, for an element $J$ of $B$ contained in $e(I)$ such that $\widehat{f}^{n m}(J)=e(I)$, we have at least two completely different (if we look at the lifting) choices. Since the length of $G^{m}(I)$ is larger than 3, this interval contains at least two different integer translations of $I$. Thus, we can choose $J$ in such a way that $G^{m}\left(I \cap e^{-1}(J)\right)$ is equal to the translation of $I$ by a non-zero integer. Thus, we will have in $J$ a fixed point of $f^{n m}$ with non-zero rotation number (for the lifting $G^{m}$ ), while the rotation numbers of all fixed points contained in $e\left(S_{i}\right)$ for $i=1, \ldots, s^{m}$ are equal to zero. As we noticed in the second paragraph of this proof, this implies that the horseboot $W^{\prime}$ is lost.

The horseboot $W^{\prime}$ is an $\left(s^{m}+1\right)$-horseboot. We have $(1 / n m) \log \left(s^{m}+1\right)>$ $(1 / n m) \log s^{m}=(1 / n) \log n$, so the proof of the lemma is complete.

At last we can prove the main theorem of this section.

Theorem 6.9. Let $f: X \rightarrow X$ be a continuous circle map of degree 1 . If $h_{l}(f, \varphi)>$ 0 , then

$$
h_{l}(f, \varphi)=\sup \left\{\frac{1}{n} \log s: f^{n} \text { has a lost s-horseboot }\right\} .
$$

Proof. If the rotation set of $f$ consists of more than one point then there are no lost measures, so $h_{l}(f, \varphi)=0$. Thus, if $h_{l}(f, \varphi)>0$ then the rotation set of $f$ consists of more than one point and we can use Lemmata $6.2-6.8$.

By Lemma 6.7 the left-hand side of (6.4) is greater than or equal to the righthand side. By Lemma $6.5 h(\widehat{f}) \geq h_{l}(\widehat{f}, \varphi)=h_{l}(f, \varphi)$. Thus, by Theorem 6.1 (for $\widehat{f})$ and Lemma 6.8 , for every $\varepsilon>0$ there exist $s$ and $n$ such that $\widehat{f}^{n}$ has a lost $s$-horseboot and $(1 / n) \log s \geq h_{l}(f, \varphi)-\varepsilon$. This horseboot can be lifted (via $\pi^{-1}$ ) to an $s$-horseboot for $f$. Since $\pi$ preserves rotation numbers, this horseboot is also lost. This proves the converse inequality in (6.4).

As a bonus, we obtain a nice result for a special class of circle maps.

Theorem 6.10. Let $f: X \rightarrow X$ be a continuous circle map of degree 1 which is leo and has rotation set consisting of more than one point. Then $h_{l}(f, \varphi)=h(f)$.

Proof. In this situation we have $\pi$ equal to the identity, that is $\widehat{f}=f$. Then the theorem follows immediately from Theorem 6.1, Lemma 6.8 and Lemma 6.7.

Let us look now at the directional entropy and directional horseboots. We will prove that if the rotation interval of $f$ is non-degenerate, then it never happens that all directional entropies are realized on directional horseboots in corresponding directions. Namely, we will show that there are irrational directions with positive 
directional entropy. On the other hand, if there is a directional horseboot of direction $v$, then there are periodic orbits of direction $v$, so $v$ is rational.

Lemma 6.11. Let $f: X \rightarrow X$ be a continuous circle map of degree 1 which has rotation set consisting of more than one point. Then $h_{l}(f, \varphi)>0$.

Proof. As we noticed before Lemma 6.4, the rotation sets of $f$ and $\widehat{f}$ are equal. Therefore the rotation set of $\widehat{f}$ consists of more than one point. Hence, $h(\widehat{f})>0$ (see e.g. $[\mathrm{ALM}]$ ). Since $\widehat{f}$ is leo, by Theorem 6.10 , the lost entropy of $\widehat{f}$ is positive, so by Lemma 6.5 , the lost entropy of $f$ is positive.

Proposition 6.12. Let $f: X \rightarrow X$ be a continuous circle map of degree 1 which has rotation set consisting of more than one point. Then there are irrational directions in which the directional entropy of $f$ is positive.

Proof. By Lemma 6.11 and Theorem 6.9 there are $n \geq 1$ and $s \geq 2$ such that $f^{n}$ has a lost $s$-horseboot $W$. Using Lemma 6.6 we pass to the full shift on $s$ symbols $\left(\Sigma_{s}, \sigma\right)$ and a function $\varphi_{\sigma}$ constant on the cylinders of length 2 (that is, arrows of the corresponding Markov graph). We can treat these arrows as vertices of a new Markov graph $G$, and in such a way we obtain a subshift of finite type, with the corresponding observable constant on the cylinders of length 1 . This system (including the observable) is conjugate to $\left(\Sigma_{s}, \sigma, \varphi_{\sigma}\right)$ and is mixing. As we noticed in the proof of Lemma 6.7, its rotation set consists of more than one point. Thus, the situation is as in Section 5.

Using the same construction as at the beginning of the proof of Proposition 5.4, we can find two loops in $G$ with different rotation numbers $a$ and $b$, the same length $n$, and passing through the same vertex $A$. If we take only the paths that are repetitions of these loops, we get the full 2-shift for $\sigma^{n}$. Bernoulli shifts supported by this 2-shift, with weights $p$ and $1-p$, where $p \in(0,1)$, give us ergodic measures for $\sigma$ with all rotation numbers between $a$ and $b$, in particular all irrational numbers from that interval. These measures have positive entropy. By Corollary 5.2, directional entropies in these directions are positive.

\section{REFERENCES}

[ALM] Ll. Alsedà, J. Llibre and M. Misiurewicz, Combinatorial Dynamics and Entropy in Dimension One, World Scientific, Singapore, 1993. MR 95j:58042

[BM] A. Blokh and M. Misiurewicz, New order for periodic orbits of interval maps, Ergod. Th. \& Dynam. Sys. 17 (1997), 565-574. MR 98i:58191

[Bot] F. Botelho, Rotational entropy for annulus endomorphisms, Pacific J. Math. 151 (1991), 1-19. MR 92i:58099

[Bow] R. Bowen, Topological entropy for noncompact sets, Trans. Amer. Math. Soc. 184 (1973), 125-136. MR 49:3082

[DGS] M. Denker, C. Grillenberger and K. Sigmund, Ergodic Theory on Compact Spaces, Lecture Notes in Math., vol. 527, Springer Verlag, Berlin, 1976. MR 56:15879

$[\mathrm{Kw}] \quad$ J. Kwapisz, Rotation sets and entropy, PhD Thesis, SUNY at Stony Brook, 1995.

[KS] J. Kwapisz and R. Swanson, Asymptotic entropy, periodic orbits, and pseudo-Anosov maps, Ergod. Th. \& Dynam. Sys. 18 (1998), 425-439. CMP 98:12

[LM] J. Llibre and M. Misiurewicz, Horseshoes, entropy and periods for graph maps, Topology 32 (1993), 649-664. MR 94k:58113

[MT] B. Marcus and S. Tuncel, The weight-per-symbol polytope and scaffolds of invariants associated with Markov chains, Ergod. Th. \& Dynam. Sys. 11 (1991), 129-180. MR 92g:28038

[M] M. Misiurewicz, Horseshoes for mappings of the interval, Bull. Acad. Pol. Sci., Sér. Sci. Math., Astr. et Phys. 27 (1979), 167-169. MR 81b:58033 
[MS] M. Misiurewicz and W. Szlenk, Entropy of piecewise continuous interval maps, Studia Math. 67 (1980), 45-63. MR 82a:58030

[PP] Y. Pesin and B. Pitskel, Topological pressure and the variational principle for noncompact sets, Functional Anal. Appl. 18 (1984), 307-318. MR 86i:28031

[Sw] R. Swanson, Periodic orbits and the continuity of rotation numbers, Proc. Amer. Math. Soc. 117 (1993), 269-273. MR 93c:58170

[Z] K. Ziemian, Rotation sets for subshifts of finite type, Fund. Math. 146 (1995), 189-201. MR 96b: 58072

Department of Mathematical Sciences, iUpui, 402 N. Blackford Street, IndianapoLIS, INDIANA 46202-3216

E-mail address: wgeller@math.iupui.edu

E-mail address: mmisiure@math.iupui.edu 